Article

\title{
The Duality between Corings and Ring Extensions
}

\section{Florin F. Nichita ${ }^{1, *}$ and Bartosz Zieliński ${ }^{2}$}

${ }^{1}$ Institute of Mathematics of the Romanian Academy, 21 Calea Grivitei Street, 010702 Bucharest, Romania

${ }^{2}$ Department of Theoretical Physics and Computer Science, University of Łódź, Pomorska 149/153, 90-236, Łódź, Poland; E-Mail: bzielinski@ uni.lodz.pl

* Author to whom correspondence should be addressed; E-Mail: florin.nichita@imar.ro; Tel.: +40-0-21-319-65-06; Fax: +40-0-21-319-65-05.

Received: 29 June 2012; in revised form: 24 July 2012 / Accepted: 30 July 2012 /

Published: 10 August 2012

\begin{abstract}
We study the duality between corings and ring extensions. We construct a new category with a self-dual functor acting on it, which extends that duality. This construction can be seen as the non-commutative case of another duality extension: the duality between finite dimensional algebras and coalgebra. Both these duality extensions have some similarities with the Pontryagin-van Kampen duality theorem.
\end{abstract}

Keywords: corings; ring extension; duality; Yang-Baxter equation

Classification: MSC 16T25; 16T15

\section{Introduction}

Non-commutative geometry is a branch of mathematics concerned with geometric approach to non-commutative algebras, and with constructions of spaces which are locally presented by non-commutative algebras of functions. Its main motivation is to extend the commutative duality between spaces and functions to the non-commutative setting.

More specifically, in topology, compact Hausdorff topological spaces can be reconstructed from the Banach algebra of functions on the space. The Pontryagin duality theorem refers to the duality between the category of compact Hausdorff Abelian groups and the category of discrete Abelian groups. The Pontryagin-van Kampen duality theorem extends this duality to all locally compact Hausdorff Abelian 
topological groups by including the categories of compact Hausdorff Abelian groups and discrete Abelian groups into the category of locally compact Hausdorff Abelian topological groups (see [1]). This can be illustrated by the following diagram:

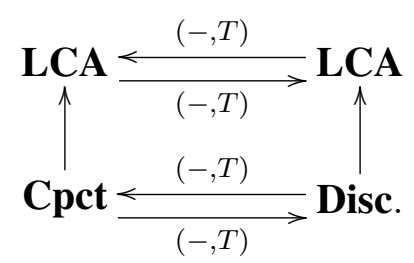

Taking the Pontryagin-van Kampen duality theorem as a model, an extension for the duality between finite dimensional algebras and coalgebras to the category of finite dimensional Yang-Baxter structures was constructed in [2]. The resulting duality theorem can be illustrated by the following diagram:

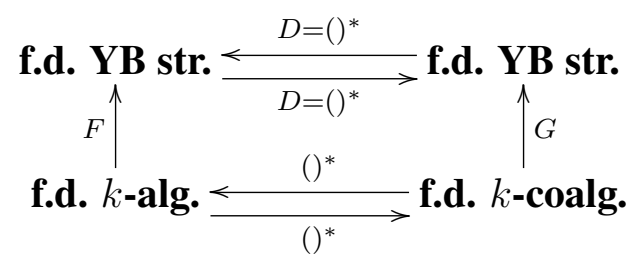

Our motivation in this paper is to extend the above duality to the non-commutative setting.

In Section 2, we present in a new fashion the duality between right finitely generated projective corings and ring extensions (compare with [3]).

In Section 3, we define the category of (right finitely generated projective) generalized Yang-Baxter structures. We construct full and faithful embeddings from the categories of ring extensions and corings to the category of generalized Yang-Baxter structures. We show that taking the right dual is a duality functor in the category of right finitely generated projective generalized Yang-Baxter structures. Then we conclude that the duality between right finitely generated projective corings and ring extensions can be lifted up to the category of right finitely generated projective generalized Yang-Baxter structures.

There are some more comments to be made.

(i) We propose as a research project the investigation of other connections between the duality of (co)algebras and the Pontryagin duality. (For example, one might try to endow the (co)algebra structures with some topological structures.)

(ii) At the epistemologic level, the extension of the duality of (co)algebra structures seems to be a model for the relation between interdisciplinarity, pluridisciplinarity and transdisciplinarity (see [4]).

(iii) This paper explains that taking the dual of some objects can be seen a "continuous" process. Let us visualize this statement by considering an example from geometry. We take a triangular prism: We can see it as two parallel triangles joint by 3 segments. In total it has 5 planar geometric figures, 9 edges and 6 vertices. The geometric dual of the triangular prism has 6 planar geometric figures, 9 edges and 5 vertices. Now, one can start with a triangular prism, "shave" its corners, and then continuously deform that figure in order to obtain the geometric dual of the triangular prism. 


\section{Notations and Preliminaries}

Throughout this paper $\mathbb{K}$ is a commutative ring, and all $\mathbb{K}$-modules $M$ are such that for all $m \in M$, $2 m=0$ implies $m=0$.

Let $A, B, C$, etc. be algebras over ground commutative ring $\mathbb{K}$. Unadorned tensor product will denote the tensor product over $\mathbb{K}$. For modules $M$ in ${ }_{A} \mathcal{M}_{B}$, symbols $M^{*},{ }^{*} M,{ }^{*} M^{*}$ denote right dual, left dual and bidual of $M$, and ${ }_{A} \mathcal{M}_{B}(M, N)$ denotes the $\mathbb{K}$-module of $(A, B)$-bimodule maps $M \rightarrow N$. In what follows we shall concentrate on right dual of $M$ but similar observations can be made for the left dual as well.

For all $\phi \in{ }_{A} \mathcal{M}_{B}(M, N)$, let $\phi^{*}: N^{*} \rightarrow M^{*}$ denote the right adjoint of $\phi$ i.e., $\phi^{*}(g)(m):=g \circ \phi(m)$.

We denote by $(\cdot)^{\text {op }}: A \rightarrow A^{\text {op }}$ the canonical anti-algebra isomorphism from the algebra $A$ into its opposite $A^{\mathrm{op}}$ (which is the identity on the underlying $\mathbb{K}$-modules), i.e., $a=a^{\mathrm{op}}$ as module elements and $\left(a a^{\prime}\right)^{\mathrm{op}}=a^{\mathrm{op}} a^{\mathrm{op}}$ for all $a, a^{\prime} \in A$.

The following facts are well known, but we recall them to set up the notation:

(i) If $M \in{ }_{A} \mathcal{M}_{B}$ then $M^{*} \in A_{A^{\text {op }}} \mathcal{M}_{B^{\text {op }}}$ with $\left(a^{\text {op }} f b^{\text {op }}\right)(m)=b f(a m)$.

Assume that $M \in{ }_{A} \mathcal{M}_{B}$ is also finitely generated projective as a right $B$-module, i.e., there exists a dual basis $\hat{f}_{i} \in M^{*}, \hat{m}_{i} \in M, i \in I$ such that for any $m \in M, m=\sum_{i} \hat{m}_{i} \hat{f}_{i}(m)$. Then

(ii) The mapping $\kappa_{M}: M \rightarrow M^{* *}, \kappa_{M}(m)(f)=f(m)^{\text {op }}$ is an isomorphism in ${ }_{A} \mathcal{M}_{B}$, with the inverse $\kappa_{M}^{-1}(\tilde{m})=\sum_{i} \hat{m}_{i} \tilde{m}\left(\hat{f}_{i}\right)^{\text {op }}$. In fact $\kappa$ is a natural morphism between identity functor in ${ }_{A} \mathcal{M}_{B}$ and the functor ()$^{* *}:{ }_{A} \mathcal{M}_{B} \rightarrow{ }_{A} \mathcal{M}_{B}$.

(iii) If $N \in{ }_{B} \mathcal{M}_{C}$ then $\kappa_{M, N}: M^{*} \otimes_{B_{\text {op }}} N^{*} \rightarrow\left(M \otimes_{B} N\right)^{*}$, given by $\kappa_{M, N}\left(f \otimes_{B_{\text {op }}} g\right)(m \otimes n)=$ $g(f(m) n)$, is an isomorphism in ${ }_{A^{\text {op }}} \mathcal{M}_{C^{\text {op }}}$ with the inverse

$$
\kappa_{M, N}^{-1}(\alpha)=\sum_{i} \hat{f}_{i} \otimes_{B^{\text {op }}} \alpha\left(\hat{m}_{i} \otimes_{B} \cdot\right)
$$

(iv) Let $M \in{ }_{A} \mathcal{M}_{B}, N \in{ }_{B} \mathcal{M}_{C}, P \in{ }_{C} \mathcal{M}_{D}$, where $A, B, C, D$ are algebras. Then the following diagram is commutative:

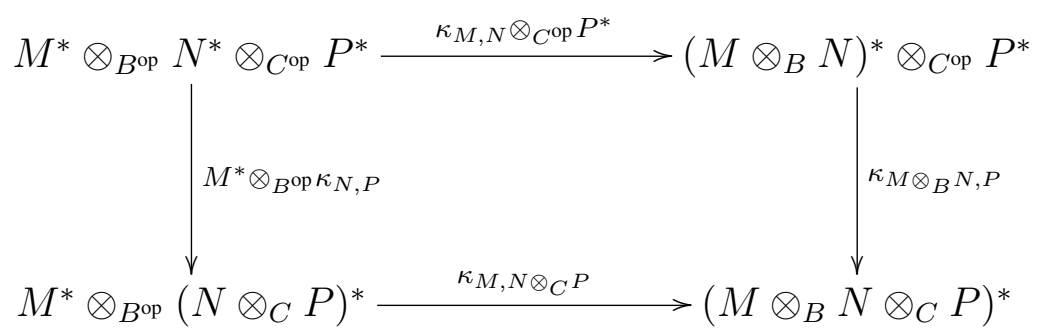

(v) Let $M \in{ }_{A} \mathcal{M}_{B}$ be finitely generated projective as $B$-module, with dual basis $\hat{m}_{i} \in M, \hat{f}_{i} \in M^{*}$, $i \in I$, and let $N \in{ }_{B} \mathcal{M}_{C}$ be finitely generated projective as a $C$-module with dual basis $\hat{n}_{i} \in N$, $\hat{g}_{i} \in N^{*}, i \in J$. Then $M \otimes_{B} N \in{ }_{A} \mathcal{M}_{C}$ is finitely generated projective as a $C$-module with a dual basis

$$
m_{i} \otimes_{B} n_{j} \in M \otimes_{B} N, \kappa_{M, N}\left(\hat{f}_{i} \otimes_{B^{\text {op }}} \hat{g}_{j}\right) \in\left(M \otimes_{B} N\right)^{*}, i \in I, j \in J
$$


The following terminology and theorems concerning corings and ring extensions are needed in this paper. For a review on coalgebras see: [5-7]. For a review on corings see [3].

Definition 2.1 $\mathcal{C} \in{ }_{B} \mathcal{M}_{B}$ is called a $B$-coring if there exist morphisms $\Delta^{\mathcal{C}}, \varepsilon^{\mathcal{C}} \in{ }_{B} \mathcal{M}_{B}, \Delta^{\mathcal{C}}: \mathcal{C} \rightarrow$ $\mathcal{C} \otimes_{B} \mathcal{C}, \varepsilon^{\mathcal{C}}: \mathcal{C} \rightarrow B$ such that

$$
\begin{gathered}
\left(\Delta^{\mathcal{C}} \otimes_{B} \mathcal{C}\right) \circ \Delta^{\mathcal{C}}=\left(\mathcal{C} \otimes_{B} \Delta^{\mathcal{C}}\right) \circ \Delta^{\mathcal{C}} \\
\left(\varepsilon^{\mathcal{C}} \otimes_{B} \mathcal{C}\right) \circ \Delta^{\mathcal{C}}=\mathcal{C}=\left(\mathcal{C} \otimes_{B} \varepsilon^{\mathcal{C}}\right) \circ \Delta^{\mathcal{C}}
\end{gathered}
$$

In the sequel we shall use Sweedler's notation $\Delta^{\mathcal{C}}(c)=c_{(1)} \otimes_{B} c_{(2)}$. Given $B$-corings $\mathcal{C}$ and $\mathcal{D}$, a map $\phi \in{ }_{B} \mathcal{M}_{B}(\mathcal{C}, \mathcal{D})$ is called a morphism of $B$-corings if $\left(\phi \otimes_{B} \phi\right) \circ \Delta^{\mathcal{C}}=\Delta^{\mathcal{D}} \circ \phi$ and $\varepsilon^{\mathcal{D}} \circ \phi=\varepsilon^{\mathcal{C}}$. The category of $B$-corings is denoted by $\mathbf{C r g}_{B}$.

Definition 2.2 Ring $\mathcal{R}$ is called an extension of a ring $B$ if there exists an injective unital ring morphism $\imath_{\mathcal{R}}: B \rightarrow \mathcal{R}$. Observe that $\mathcal{R} \in{ }_{B} \mathcal{M}_{B}$ by $\imath_{\mathcal{R}}$. Given ring extensions $\imath_{\mathcal{R}}: B \rightarrow \mathcal{R}$ and $\imath_{\mathcal{P}}: B \rightarrow \mathcal{P}$, a ring morphism $\alpha: \mathcal{R} \rightarrow \mathcal{P}$ is called a morphism of ring extensions if $\alpha \circ \imath_{\mathcal{R}}=\imath_{\mathcal{P}}$ or, equivalently, if $\alpha \in{ }_{B} \mathcal{M}_{B}(\mathcal{R}, \mathcal{P})$. The category of ring extensions of $B$ is denoted by $\mathbf{R g e}_{B}$.

The full subcategory of $\mathbf{C r g}_{B}$ (resp. Rge $_{B}$ ) consisting of those $B$-corings (resp. ring extensions of $B$ ) that are finitely generated projective as right $B$-modules is denoted by r.f.g.pCrg (resp. r.f.g.pRge $_{B}$ ).

Lemma 2.3 (i) If $\mathcal{C} \in \mathbf{C r g}_{B}$ then $\mathcal{C}^{*} \in{ }_{B^{o p}} \mathcal{M}_{B^{o p}}$ is a ring extension of $B^{\text {op }}$ with multiplication

$$
\left(r r^{\prime}\right)(c):=r^{\prime}\left(r\left(c_{(1)}\right) c_{(2)}\right), \quad \text { for all } r, r^{\prime} \in \mathcal{C}^{*}
$$

unit $1_{\mathcal{C}^{*}}:=\varepsilon^{\mathcal{C}}$ and embedding map

$$
\imath_{\mathcal{C}^{*}}: B \rightarrow \mathcal{C}^{*}, \quad b^{o p} \mapsto b^{o p} 1_{\mathcal{C}^{*}}
$$

(ii) If $\phi: \mathcal{C} \rightarrow \mathcal{D}$ is any coring morphism then $\phi^{*}: \mathcal{D}^{*} \rightarrow \mathcal{C}^{*}$ is a ring extension morphism.

(iii) If $\mathcal{R} \in$ r.f.g.pRge ${ }_{B}$ then $\mathcal{R}^{*}$ is a $B^{o p}$-coring with comultiplication and counit

$$
\begin{gathered}
\Delta^{\mathcal{R}^{*}}: \mathcal{R}^{*} \rightarrow \mathcal{R}^{*} \otimes_{B^{o p}} \mathcal{R}^{*} c \mapsto \sum_{i} \hat{f}_{i} \otimes_{B^{o p}} c\left(\hat{r}_{i} \cdot\right) \\
\varepsilon^{\mathcal{R}^{*}}: \mathcal{R}^{*} \rightarrow B^{o p}, \quad c \mapsto c\left(1_{\mathcal{R}}\right)^{o p}
\end{gathered}
$$

where $\hat{r}_{i} \in \mathcal{R}, \hat{f}_{i} \in \mathcal{R}^{*}, i \in I$ is a (finite) dual basis of $\mathcal{R}$.

(iv) If $\phi: \mathcal{R} \rightarrow \mathcal{S}$ is a morphism of right finitely generated projective ring extensions of $B$, then $\phi: \mathcal{S}^{*} \rightarrow \mathcal{R}^{*}$ is a morphism of $B^{o p}$-corings.

(v) Functor ( $)^{* *}:$ r.f.g.pRge . $_{B} \rightarrow$ r.f.g.pRge ${ }_{B}$ is equivalent to the identity functor on r.f.g.pRge . For all $\mathcal{R} \in$ r.f.g.pRge ${ }_{B}, \kappa_{\mathcal{R}}: \mathcal{R} \rightarrow \mathcal{R}^{* *}$ is a ring extension isomorphism facilitating this equivalence.

(vi) Functor ()** : r.f.g.pCrg ${ }_{B} \rightarrow$ r.f.g.pCrg ${ }_{B}$ is equivalent to the identity functor on r.f.g.pCrg . For $^{*}$ all $\mathcal{C} \in$ r.f.g.pCrg ${ }_{B}, \kappa_{\mathcal{C}}: \mathcal{C} \rightarrow \mathcal{C}^{* *}$ is a B-coring isomorphism facilitating this equivalence.

Proof. The statements (i) and (ii) are contained in Proposition 3.2 [8], while (iii) and (v) are rephrasings of Theorem 3.7 [8] (cf. [3], 17.8-17.13) 
(iv) Consider any ring extension morphism $\phi: \mathcal{R} \rightarrow \mathcal{S}$. Let $\hat{r}_{i} \in \mathcal{R}, \hat{f}_{i} \in \mathcal{R}^{*}, i \in I$ be any finite dual basis of $\mathcal{R}$, and let $\hat{s}_{i} \in \mathcal{S}, \hat{g}_{i} \in \mathcal{S}^{*}, i \in J$ be any finite dual basis of $\mathcal{S}$. For all $s \in \mathcal{S}^{*}$,

$$
\begin{aligned}
& \Delta^{\mathcal{R}^{*}} \circ \phi^{*}(s)= \sum_{i} \hat{f}_{i} \otimes_{B^{\text {op }}} s\left(\phi\left(\hat{r}_{i}\right) \phi(\cdot)\right)=\sum_{i j} \hat{f}_{i} \otimes_{B^{\text {op }}} s\left(\hat{s}_{j} \hat{g}_{j}\left(\phi\left(\hat{r}_{i}\right)\right) \phi(\cdot)\right) \\
&= \sum_{i j} \hat{f}_{i} \otimes_{B^{\text {op }}} s\left(\hat{s}_{j} \phi\left(\hat{g}_{j}\left(\phi\left(\hat{r}_{i}\right)\right) \cdot\right)\right)=\sum_{i j} \hat{f}_{i} \otimes_{B^{\text {op }}} \hat{g}_{j}\left(\phi\left(\hat{r}_{i}\right)\right)^{\mathrm{op}} s\left(\hat{s}_{j} \phi(\cdot)\right) \\
&=\sum_{i j} \hat{f}_{i} \hat{g}_{j}\left(\phi\left(\hat{r}_{i}\right)\right)^{\mathrm{op}} \otimes_{B^{\text {op }}} s\left(\hat{s}_{j} \phi(\cdot)\right)=\sum_{j} \hat{g}_{j} \circ \phi \otimes_{B^{\text {op }}} s\left(\hat{s}_{j} \phi(\cdot)\right) \\
&=\left(\phi^{*} \otimes_{B^{\text {op }}} \phi^{*}\right)\left(\sum_{j} \hat{g}_{j} \otimes_{B^{\text {op }}} s\left(\hat{s}_{j} \cdot\right)\right)=\left(\phi^{*} \otimes_{B^{\text {op }}} \phi^{*}\right) \circ \Delta^{\mathcal{S}}(s)
\end{aligned}
$$

and

$$
\varepsilon^{\mathcal{R}^{*}} \circ \phi^{*}(s)=\varepsilon^{\mathcal{R}^{*}}(s \circ \phi)=(s \circ \phi)\left(1_{\mathcal{R}}\right)=s\left(1_{\mathcal{S}}\right)=\varepsilon^{\mathcal{S}^{*}}(s)
$$

Hence $\phi^{*}$ is a coring map.

(vi) It is enough to prove that $\kappa_{\mathcal{C}}$, is a coring map for any $\mathcal{C} \in$ r.f.g.p $\operatorname{Crg}_{B}$. Let $\mathcal{C}$ be a $B$-coring, and let $\hat{c}_{i} \in \mathcal{C}, \hat{f}_{i} \in \mathcal{C}^{*}, i \in I$, be any finite dual basis of $\mathcal{C}$. Observe that $\hat{f}_{i} \in \mathcal{C}^{*}, \kappa_{\mathcal{C}}(\hat{c}) \in \mathcal{C}^{* *}, i \in I$ is a dual basis of $\mathcal{C}^{*}$. Indeed, for any $g \in \mathcal{C}^{*}$,

$$
g=\sum_{i} \hat{f}_{i} g\left(\hat{m}_{i}\right)^{\mathrm{op}}=\sum_{i} \hat{f}_{i} \kappa_{\mathcal{C}}\left(\hat{m}_{i}\right)
$$

Hence, for all $c \in \mathcal{C}$,

$$
\begin{aligned}
& \Delta^{\mathcal{C}^{* *}} \circ \kappa_{\mathcal{C}}(c)= \sum_{i} \kappa_{\mathcal{C}}\left(\hat{c}_{i}\right) \otimes_{B} \kappa_{\mathcal{C}}(c)\left(\hat{f}_{i} \cdot\right)=\sum_{i} \kappa_{\mathcal{C}}\left(\hat{c}_{i}\right) \otimes_{B}\left(\hat{f}_{i} \cdot\right)(c)^{\mathrm{op}} \\
&=\sum_{i} \kappa_{\mathcal{C}}\left(\hat{c}_{i}\right) \otimes_{B} \cdot\left(\hat{f}_{i}\left(c_{(1)}\right) c_{(2)}\right)^{\mathrm{op}}=\sum_{i} \kappa_{\mathcal{C}}\left(\hat{c}_{i}\right) \otimes_{B} \kappa_{\mathcal{C}}\left(\hat{f}_{i}\left(c_{(1)}\right) c_{(2)}\right) \\
&=\sum_{i} \kappa_{\mathcal{C}}\left(\hat{c}_{i} \hat{f}_{i}\left(c_{(1)}\right) \otimes_{B} \kappa_{\mathcal{C}}\left(c_{(2)}\right)=\left(\kappa_{\mathcal{C}} \otimes_{B} \kappa_{\mathcal{C}}\right) \circ \Delta^{\mathcal{C}}(c)\right.
\end{aligned}
$$

and

$$
\varepsilon^{\mathcal{C}^{* *}} \circ \kappa_{\mathcal{C}}(c)=\kappa_{\mathcal{C}}(c)\left(1_{\mathcal{C}^{*}}\right)^{\mathrm{op}}=1_{\mathcal{C}^{*}}(c)^{\mathrm{opop}}=\varepsilon^{\mathcal{C}}(c)
$$

Corollary 2.4()$^{*}$ is a duality functor between r.f.g.pRge $\mathbf{B}_{B}$ and $\mathbf{r . f . g . p C r g} \mathrm{B}_{B^{o p}}$ :

$$
\text { r.f.g.pRge } e_{B} \underset{()^{*}}{\longleftarrow} \text { r.f.g.pCrg }{ }_{B^{o p}}
$$

\section{An Extension for the Duality between Corings and Ring Extensions}

Our aim in this section is to extend the duality between right finitely generated projective ring extensions and corings to the category of right finitely generated projective generalized Yang-Baxter structures. 
We use the following terminology concerning the Yang-Baxter equation. Some references on this topic are: [9-11], etc.

Let $B$ be a $\mathbb{K}$-algebra. Given a $(B, B)$-bimodule $V$ and a $(B, B)$-bilinear map $R: V \otimes_{B} V \rightarrow V \otimes_{B} V$ we write $R^{12}=R \otimes_{B} i d, R^{23}=i d \otimes_{B} R: V \otimes_{B} V \otimes_{B} V \rightarrow V \otimes_{B} V \otimes_{B} V$ where $i d: V \rightarrow V$ is the identity map.

Definition 3.1 An invertible $(B, B)$-linear map $R: V \otimes_{B} V \rightarrow V \otimes_{B} V$ is called a generalized Yang-Baxter operator (or simply a generalised $Y B$ operator) if it satisfies the equation

$$
R^{12} \circ R^{23} \circ R^{12}=R^{23} \circ R^{12} \circ R^{23}
$$

Definition 3.2 For an algebra $B$, we define the category $\mathbf{Y B} \operatorname{str}_{B}$ whose objects are 4-tuples $(V, \varphi, e, \varepsilon)$, where

(i) $V$ is a $(B, B)$-bimodule;

(ii) $\varphi: V \otimes_{B} V \rightarrow V \otimes_{B} V$ is a generalized YB operator;

(iii) $e \in V$ such that for all $b \in B$, $e b=b e$, and for all $x \in V, \varphi(x \otimes e)=e \otimes_{B} x, \varphi\left(e \otimes_{B} x\right)=x \otimes_{B} e$;

(iv) $\varepsilon: V \rightarrow B$ is a $(B, B)$-bimodule map, such that $\left(i d \otimes_{B} \varepsilon\right) \circ \varphi=\varepsilon \otimes_{B} i d,\left(\varepsilon \otimes_{B} i d\right) \circ \varphi=i d \otimes_{B} \varepsilon$.

A morphism $f:(V, \varphi, e, \varepsilon) \rightarrow\left(V^{\prime}, \varphi^{\prime}, e^{\prime}, \varepsilon^{\prime}\right)$ in the category $\mathbf{Y B} \operatorname{str}_{B}$ is a $(B, B)$-bilinear map $f: V \rightarrow V^{\prime}$ such that:

(v) $\left(f \otimes_{B} f\right) \circ \varphi=\varphi^{\prime} \circ\left(f \otimes_{B} f\right)$,

(vi) $f(e)=e^{\prime}$,

(vii) $\varepsilon^{\prime} \circ f=\varepsilon$.

Composition of morphisms is defined as the standard composition of $B$-linear maps. A full subcategory

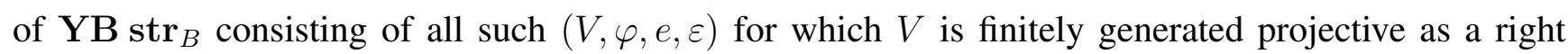
$B$-module is defined by r.f.g.pYB $\operatorname{str}_{B}$.

Remark 3.3 Let $R: V \otimes_{B} V \rightarrow V \otimes_{B} V$ be a generalised YB operator . Then $(V, R, 0,0)$ is an object in the category $\mathrm{YB} \mathbf{s t r}_{B}$.

Theorem 3.4 (i) There exists a functor:

$$
\begin{aligned}
& F: \mathbf{R g e}_{B} \rightarrow \mathbf{Y B} \operatorname{str}_{B}, \quad \mathcal{R} \mapsto\left(\mathcal{R}, \varphi_{\mathcal{R}}, 1_{\mathcal{R}}, 0 \in{ }^{*} \mathcal{R}^{*}\right) \\
& \text { where } \varphi_{\mathcal{R}}\left(r \otimes_{B} r^{\prime}\right)=r r^{\prime} \otimes_{B} 1+1 \otimes_{B} r r^{\prime}-r \otimes_{B} r^{\prime}
\end{aligned}
$$

Any ring extension map $f$ is simply mapped into a $(B, B)$ bimodule map.

(ii) $F$ is a full and faithful embedding.

Proof. i) The proof that $\varphi_{\mathcal{R}}$ is a generalised YB operator is left to the reader (cf. Proposition 2.1 from [12], $\left.\varphi_{\mathcal{R}}^{-1}=\varphi_{\mathcal{R}}\right)$. Furthermore $\varphi_{\mathcal{R}}\left(r \otimes_{B} 1\right)=r \otimes_{B} 1+1 \otimes_{B} r-r \otimes_{B} 1=1 \otimes_{B} r, \varphi_{\mathcal{R}}\left(1 \otimes_{B} r\right)=$ $1 \otimes_{B} r+r \otimes_{B} 1-1 \otimes_{B} r=r \otimes_{B} 1,\left(i d \otimes_{B} 0\right) \circ \varphi_{\mathcal{R}}=0=\left(0 \otimes_{B} i d\right),\left(0 \otimes_{B} i d\right) \circ \varphi_{\mathcal{R}}=0=\left(i d \otimes_{B} 0\right)$. Hence $\left(\mathcal{R}, \varphi_{\mathcal{R}}, 1_{\mathcal{R}}, 0\right)$ is an object in the category $\mathbf{Y B} \mathbf{s t r}_{B}$. 
Let $f: \mathcal{R} \rightarrow \mathcal{S}$ be a morphism of ring extensions. Then $f\left(1_{\mathcal{R}}\right)=1_{\mathcal{S}}$ and $0 \circ f=0$. Moreover

$$
\begin{aligned}
\left(f \otimes_{B} f\right) \circ \varphi_{\mathcal{R}}\left(r \otimes_{B} r^{\prime}\right)=f(r) f\left(r^{\prime}\right) \otimes_{B} f(1)+f(1) \otimes_{B} f(r) f\left(r^{\prime}\right)-f(r) \otimes_{B} f\left(r^{\prime}\right) & =\varphi_{\mathcal{S}} \circ\left(f \otimes_{B} f\right)\left(r \otimes_{B} r^{\prime}\right) .
\end{aligned}
$$

Hence $f:\left(\mathcal{R}, \varphi_{\mathcal{R}}, 1_{\mathcal{R}}, 0\right) \rightarrow\left(\mathcal{S}, \varphi_{\mathcal{S}}, 1_{\mathcal{S}}, 0\right)$ is a morphism in the category $\mathbf{Y B} \operatorname{str}_{B}$.

(ii) If $F \mathcal{R}=F \mathcal{S}$, for some $\mathcal{R}, \mathcal{S} \in \mathbf{R g e}_{B}$, then obviously $\mathcal{R}=\mathcal{S}$ as $(B, B)$-bimodules, $1_{\mathcal{S}}=1_{\mathcal{R}}$, and the only thing which can differ is the multiplication. Denote by $\cdot$ the multiplication in $\mathcal{R}$, and by $\circ$ the multiplication in $\mathcal{S}$. Then, as $\varphi_{\mathcal{R}}=\varphi_{\mathcal{S}}$, for all $r, r^{\prime} \in \mathcal{R}$,

$$
r \cdot r^{\prime} \otimes_{B} 1+1 \otimes_{B} r \cdot r^{\prime}-r \otimes_{B} r^{\prime}=r \circ r^{\prime} \otimes_{B} 1+1 \otimes_{B} r \circ r^{\prime}-r \otimes_{B} r^{\prime}
$$

hence

$$
\left(r \cdot r^{\prime}-r \circ r^{\prime}\right) \otimes_{B} 1=-1 \otimes_{B}\left(r \cdot r^{\prime}-r \circ r^{\prime}\right)
$$

Multiplying tensor factors on both sides of this equation (whether using multiplication in $\mathcal{R}$ or $\mathcal{S}$ is irrelevant) yields $2\left(r \cdot r^{\prime}-r \circ r^{\prime}\right)=0$, hence $r \cdot r^{\prime}=r \circ r^{\prime}$, and so $\mathcal{R}=\mathcal{S}$ as algebras. Therefore $F$ is an embedding.

Obviously, distinct ring extension maps are also distinct as $(B, B)$-bimodule morphisms, hence $F$ is a faithful functor.

Let $f:\left(\mathcal{R}, \varphi_{\mathcal{R}}, 1_{\mathcal{R}}, 0\right) \rightarrow\left(\mathcal{S}, \varphi_{\mathcal{S}}, 1_{\mathcal{S}}, 0\right)$ be a morphism in YB $\operatorname{str}_{B}$, where $\mathcal{R}, \mathcal{S} \in \mathbf{R g e}_{B}$. Then $f$ is unital, and $\left(f \otimes_{B} f\right) \circ \varphi_{\mathcal{R}}=\varphi_{\mathcal{S}} \circ\left(f \otimes_{B} f\right)$, hence, for all $r, r^{\prime} \in \mathcal{R}$,

$$
f\left(r r^{\prime}\right) \otimes_{B} 1+1 \otimes_{B} f\left(r r^{\prime}\right)-f(r) \otimes_{B} f\left(r^{\prime}\right)=f(r) f\left(r^{\prime}\right) \otimes_{B} 1+1 \otimes_{B} f(r) f\left(r^{\prime}\right)-f(r) \otimes_{B} f\left(r^{\prime}\right) .
$$

Multiplying factors in tensor products in both sides of the above equation yields $2\left(f\left(r r^{\prime}\right)-f(r) f\left(r^{\prime}\right)\right)=$ 0 , hence $f\left(r r^{\prime}\right)=f(r) f\left(r^{\prime}\right)$ and, as $f$ is a $(B, B)$-bimodule map, it is a ring extension map. Therefore, $F$ is a full functor.

\section{Theorem 3.5 (i) There exists a functor}

$$
\begin{gathered}
G: \mathbf{C r g}_{B} \rightarrow \mathbf{Y B} \operatorname{str}_{B}, \quad \mathcal{C} \mapsto\left(\mathcal{C}, \psi_{\mathcal{C}}, 0, \varepsilon^{\mathcal{C}}\right) \\
\text { where } \psi_{\mathcal{C}}=\Delta^{\mathcal{C}} \otimes_{B} \varepsilon^{\mathcal{C}}+\varepsilon^{\mathcal{C}} \otimes_{B} \Delta^{\mathcal{C}}-i d \otimes_{B} i d
\end{gathered}
$$

A coring morphism is mapped into a $(B, B)$-bimodule morphism.

(ii) $G$ is a full and faithful embbeding.

Proof. i) The proof that $\psi_{\mathcal{C}}$ is a generalised YB operator (cf. Proposition 2.3 from [12]) is left to the reader $\left(\psi_{\mathcal{C}}^{-1}=\psi_{\mathcal{C}}\right)$. Furthermore, for all $c \in \mathcal{C}, \psi_{\mathcal{C}}\left(c \otimes_{B} 0\right)=0=0 \otimes_{B} c, \psi_{\mathcal{C}}\left(0 \otimes_{B} c\right)=0=c \otimes_{B} 0$. Moreover, for all $c, c^{\prime} \in \mathcal{C}$,

$$
\begin{aligned}
\left(i d \otimes_{B} \varepsilon^{\mathcal{C}}\right) \circ \psi_{\mathcal{C}}\left(c \otimes_{B} c^{\prime}\right)=c_{(1)} \varepsilon^{\mathcal{C}}\left(c_{(2)}\right) \varepsilon^{\mathcal{C}}\left(c^{\prime}\right)+\varepsilon^{\mathcal{C}}(c) c^{\prime}{ }_{(1)} \mathcal{\varepsilon}^{\mathcal{C}}\left(c^{\prime}{ }_{(2)}\right)-c \varepsilon^{\mathcal{C}}\left(c^{\prime}\right) & =\varepsilon^{\mathcal{C}}(c) c^{\prime} \\
& =\left(\varepsilon^{\mathcal{C}} \otimes_{B} i d\right)\left(c \otimes_{B} c^{\prime}\right)
\end{aligned}
$$


and

$$
\begin{aligned}
\left(\varepsilon^{\mathcal{C}} \otimes_{B} i d\right) \circ \psi_{\mathcal{C}}\left(c \otimes_{B} c^{\prime}\right)=\varepsilon^{\mathcal{C}}\left(c_{(1)}\right) c_{(2)} \varepsilon^{\mathcal{C}}\left(c^{\prime}\right)+\varepsilon^{\mathcal{C}}(c) \varepsilon^{\mathcal{C}}\left(c^{\prime}{ }_{(1)}\right) c^{\prime}{ }_{(2)}-\varepsilon^{\mathcal{C}}(c) c^{\prime} & =c \varepsilon^{\mathcal{C}}\left(c^{\prime}\right) \\
& =\left(i d \otimes_{B} \varepsilon^{\mathcal{C}}\right)\left(c \otimes_{B} c^{\prime}\right)
\end{aligned}
$$

Hence $\left(\mathcal{C}, \psi_{\mathcal{C}}, 0, \varepsilon^{\mathcal{C}}\right)$ is an object in $\mathbf{Y B} \operatorname{str}_{B}$. Let $f: \mathcal{C} \rightarrow \mathcal{D}$ be any morphism of $B$-corings. Then $f$ is also a $(B, B)$-bimodule morphism, $f(0)=0, \varepsilon^{\mathcal{D}} \circ f=\varepsilon^{\mathcal{C}}$, and,

$$
\begin{aligned}
\psi_{\mathcal{D}} \circ\left(f \otimes_{B} f\right)=\Delta^{\mathcal{D}} \circ f \otimes_{B} \varepsilon^{\mathcal{D}} \circ f+\varepsilon^{\mathcal{D}} \circ f \otimes_{B} \Delta^{\mathcal{D}} \circ f-f \otimes_{B} f \\
\quad=\left(f \otimes_{B} f\right) \circ \Delta^{\mathcal{C}} \otimes_{B} \varepsilon^{\mathcal{C}}+\varepsilon^{\mathcal{C}} \otimes_{B}\left(f \otimes_{B} f\right) \circ \Delta^{\mathcal{C}}+f \otimes_{B} f=\left(f \otimes_{B} f\right) \circ \psi_{\mathcal{C}}
\end{aligned}
$$

Therefore $f:\left(\mathcal{C}, \psi_{\mathcal{C}}, 0, \varepsilon^{\mathcal{C}}\right) \rightarrow\left(\mathcal{D}, \psi_{\mathcal{D}}, 0, \varepsilon^{\mathcal{D}}\right)$ is a morphism in YB $\operatorname{str}_{B}$.

(ii) Suppose that $G \mathcal{C}=G \mathcal{D}$ for some $B$-corings $\mathcal{C}, \mathcal{D}$. This means that $\mathcal{C}=\mathcal{D}$ as $(B, B)$-bimodules, $\varepsilon^{\mathcal{C}}=\varepsilon^{\mathcal{D}}$, and the only things which can differ are comultiplications. However, as $\psi_{\mathcal{C}}=\psi_{\mathcal{D}}$, we have

$$
\Delta^{\mathcal{C}} \otimes_{B} \varepsilon^{\mathcal{C}}+\varepsilon^{\mathcal{C}} \otimes_{B} \Delta^{\mathcal{C}}-I \otimes_{B} I=\Delta^{\mathcal{D}} \otimes_{B} \varepsilon^{\mathcal{C}}+\varepsilon^{\mathcal{C}} \otimes_{B} \Delta^{\mathcal{D}}-I \otimes_{B} I
$$

hence

$$
\left(\Delta^{\mathcal{C}}-\Delta^{\mathcal{D}}\right) \otimes_{B} \varepsilon^{\mathcal{C}}=-\varepsilon^{\mathcal{C}} \otimes_{B}\left(\Delta^{\mathcal{C}}-\Delta^{\mathcal{D}}\right)
$$

Composing both sides of the above equation with $\Delta^{\mathcal{C}}$ yields $2\left(\Delta^{\mathcal{C}}-\Delta^{\mathcal{D}}\right)=0$ hence $\Delta^{\mathcal{C}}=\Delta^{\mathcal{D}}$ and $\mathcal{C}=\mathcal{D}$ as $(B, B)$-corings. Hence $G$ is an embedding.

Obviously distinct $B$-coring morphisms are also distinct as $(B, B)$-bimodule morphisms, hence $G$ is a faithful functor.

Let $f:\left(\mathcal{C}, \psi_{\mathcal{C}}, 0, \varepsilon^{\mathcal{C}}\right) \rightarrow\left(\mathcal{D}, \psi_{\mathcal{D}}, 0, \varepsilon^{\mathcal{D}}\right)$, where $\mathcal{C}, \mathcal{D}$ are corings, be a morphism in YB str $_{B}$. Then $(B, B)$-bimodule morphism $f: \mathcal{C} \rightarrow \mathcal{D}$ is counital, i.e., $\varepsilon^{\mathcal{D}} \circ f=\varepsilon^{\mathcal{C}}$. Furthermore, $\left(f \otimes_{B} f\right) \circ \psi_{\mathcal{C}}=$ $\psi_{\mathcal{D}} \circ\left(f \otimes_{B} f\right)$, and hence $\left(f \otimes_{B} f\right) \circ \psi_{\mathcal{C}} \circ \Delta^{\mathcal{C}}=\psi_{\mathcal{D}} \circ\left(f \otimes_{B} f\right) \circ \Delta^{\mathcal{C}}$. Observe that $\psi_{\mathcal{C}} \circ \Delta^{\mathcal{C}}=\Delta^{\mathcal{C}}$. Therefore

$$
\begin{aligned}
\left(f \otimes_{B} f\right) \circ \Delta^{\mathcal{C}}= & \left(f \otimes_{B} f\right) \circ \psi_{\mathcal{C}} \circ \Delta^{\mathcal{C}}=\psi_{\mathcal{D}} \circ\left(f \otimes_{B} f\right) \circ \Delta^{\mathcal{C}} \\
& =\left(\Delta^{\mathcal{D}} \circ f \otimes_{B} \varepsilon^{\mathcal{D}} \circ f+\varepsilon^{\mathcal{D}} \circ f \otimes_{B} \Delta^{\mathcal{D}} \circ f-f \otimes_{B} f\right) \circ \Delta^{\mathcal{C}} \\
& =\left(\Delta^{\mathcal{D}} \circ f \otimes_{B} \varepsilon^{\mathcal{C}}+\varepsilon^{\mathcal{C}} \otimes_{B} \Delta^{\mathcal{D}} \circ f-f \otimes_{B} f\right) \circ \Delta^{\mathcal{C}}=2 \Delta^{\mathcal{D}} \circ f-\left(f \otimes_{B} f\right) \circ \Delta^{\mathcal{C}}
\end{aligned}
$$

i.e., $2\left(f \otimes_{B} f\right) \circ \Delta^{\mathcal{C}}=2 \Delta^{\mathcal{D}} \circ f$, hence $\left(f \otimes_{B} f\right) \circ \Delta^{\mathcal{C}}=\Delta^{\mathcal{D}} \circ f$, and $f$ is a $B$-coring map. Therefore $G$ is full.

Proposition 3.6 Let $(V, R, e, \varepsilon) \in$ r.f.g.pYB $\operatorname{str}_{B}$. Then

$$
(V, R, e, \varepsilon)^{*}:=\left(V^{*}, R^{\dagger}, \varepsilon, e^{\dagger}\right) \in \text { r.f.g.pYB } \operatorname{str}_{B^{o p}}
$$

where $e^{\dagger}(f)=f(e)$, and

$$
R^{\dagger}=\kappa_{V, V}^{-1} \circ R^{*} \circ \kappa_{V, V}
$$

Moreover,

$$
\kappa:() \rightarrow()^{* *}, \quad \kappa_{V}:(V, R, e, \varepsilon) \rightarrow\left(V^{* *}, R^{\dagger \dagger}, e^{\dagger}, \varepsilon^{\dagger}\right)
$$

is a natural isomorphism in r.f.g.pYB $\operatorname{str}_{B}$. 
Proof. $R$ is invertible, hence $R^{\dagger-1}=\kappa_{V, V}^{-1} \circ\left(R^{-1}\right)^{*} \circ \kappa_{V, V}$. We shall prove that $R^{\dagger}$ satisfies the Yang-Baxter equation. Observe that

$$
\begin{aligned}
& \kappa_{V \otimes_{B} V, V}^{-1} \circ\left(R \otimes_{B} I\right)^{*} \circ \kappa_{V \otimes_{B} V, V}=R^{*} \otimes_{B^{\text {op }}} I \\
& \kappa_{V, V \otimes_{B} V}^{-1} \circ\left(I \otimes_{B} R\right)^{*} \circ \kappa_{V, V \otimes_{B} V}=I \otimes_{B^{\text {op }}} R^{*}
\end{aligned}
$$

Indeed, let $\Gamma \in\left(V \otimes_{B} V\right)^{*}, f \in V^{*}$, and let $\hat{v}_{i} \in V, \hat{f}_{i} \in V^{*}, i \in I$, be a dual basis of $V$.

$$
\begin{gathered}
\kappa_{V \otimes_{B} V, V}^{-1} \circ\left(R \otimes_{B} I\right)^{*} \circ \kappa_{V \otimes_{B} V, V}\left(\Gamma \otimes_{B^{\text {op }}} f\right) \\
=\kappa_{V \otimes_{B} V, V}^{-1} \circ\left(R \otimes_{B} I\right)^{*}\left(v \otimes_{B} v^{\prime} \otimes_{B} v^{\prime \prime} \mapsto f\left(\Gamma\left(v \otimes_{B} v^{\prime}\right) v^{\prime \prime}\right)\right) \\
=\kappa_{V \otimes_{B} V, V}^{-1}\left(v \otimes_{B} v^{\prime} \otimes_{B} v^{\prime \prime} \mapsto f\left(\Gamma\left(R\left(v \otimes_{B} v^{\prime}\right)\right) v^{\prime \prime}\right)\right) \\
=\sum_{i, j \in I} \kappa_{V, V}\left(\hat{f}_{i} \otimes_{B^{\text {op }}} \hat{f}_{j}\right) \otimes_{B^{\text {op }}} f\left(\Gamma\left(R\left(\hat{v}_{i} \otimes_{B} \hat{v}_{j}\right)\right) \cdot\right) \\
=\sum_{i, j \in I} \kappa_{V, V}\left(\hat{f}_{i} \otimes_{B^{\text {op }}} \hat{f}_{j}\right) \Gamma\left(R\left(\hat{v}_{i} \otimes_{B} \hat{v}_{j}\right)\right)^{\text {op }} \otimes_{B^{\text {op }}} f \\
=\left(v \otimes_{B} v^{\prime} \mapsto \sum_{i, j \in I} \Gamma\left(R\left(\hat{v}_{i} \otimes_{B} \hat{v}_{j}\right)\right) \hat{f}_{j}\left(\hat{f}_{i}(v) v^{\prime}\right)\right) \otimes_{B^{\text {op }}} f \\
=\Gamma \circ R \otimes_{B^{\text {op }}} f=\left(R^{*} \otimes_{B^{\text {op }}} I\right)\left(\Gamma \otimes_{B^{\text {op }}} f\right)
\end{gathered}
$$

Similarly we can prove the other equality. By virtue of $(17,18)$, we can write

$$
\begin{aligned}
& R^{\dagger} \otimes_{B^{\text {op }}} I=\left(\kappa_{V, V}^{-1} \otimes_{B^{\text {op }}} I\right) \circ \kappa_{V \otimes_{B} V, V}^{-1} \circ\left(R \otimes_{B} I\right)^{*} \circ \kappa_{V \otimes_{B} V, V} \circ\left(\kappa_{V, V} \otimes_{B^{\text {op }}} I\right) \\
& I \otimes_{B^{\text {op }}} R^{\dagger}=\left(I \otimes_{B^{\text {op }}} \kappa_{V, V}^{-1}\right) \circ \kappa_{V, V \otimes_{B} V}^{-1} \circ\left(I \otimes_{B} R\right)^{*} \circ \kappa_{V, V \otimes_{B} V} \circ\left(I \otimes_{B^{\text {op }}} \kappa_{V, V}\right)
\end{aligned}
$$

By (2),

$$
\begin{gathered}
\kappa_{V \otimes_{B} V, V} \circ\left(\kappa_{V, V} \otimes_{B^{\text {op }}} I\right) \circ\left(I \otimes_{B^{\text {op }}} \kappa_{V, V}^{-1}\right)=\kappa_{V, V \otimes_{B} V} \\
\kappa_{V, V \otimes_{B} V} \circ\left(I \otimes_{B^{\text {op }}} \kappa_{V, V}\right)\left(\kappa_{V, V}^{-1} \otimes_{B^{\text {op }}} I\right)=\kappa_{V \otimes_{B} V, V}
\end{gathered}
$$

and therefore

$$
\begin{aligned}
& R^{\dagger 12} R^{\dagger 23} R^{\dagger 12}=\left(\kappa_{V, V}^{-1} \otimes_{B^{\text {op }}} I\right) \circ \kappa_{V \otimes_{B} V, V}^{-1} \circ\left(R^{12} \circ R^{23} \circ R^{12}\right)^{*} \circ \kappa_{V \otimes_{B} V, V} \circ\left(\kappa_{V, V} \otimes_{B^{\text {op }}} I\right) \\
& \quad=\left(I \otimes_{B^{\text {op }}} \kappa_{V, V}^{-1}\right) \circ \kappa_{V, V \otimes_{B} V}^{-1} \circ\left(R^{23} \circ R^{12} \circ R^{23}\right)^{*} \circ \kappa_{V, V \otimes_{B} V} \circ\left(I \otimes_{B^{\text {op }}} \kappa_{V, V}\right)=R^{\dagger 23} R^{\dagger 12} R^{\dagger 23}
\end{aligned}
$$

Hence $R^{\dagger}$ is a generalised YB operator .

Proofs of bilinearity of $e^{*}$ and centrality of $\varepsilon$ are the same as proofs of analogues properties of duals of units and counits in Lemma 2.4. Moreover, for all $f \in V^{*}$,

$$
\begin{aligned}
R^{\dagger}\left(\varepsilon \otimes_{B^{\text {op }}} f\right)= & \kappa_{V, V}^{-1} \circ R^{*} \circ \kappa_{V, V}\left(\varepsilon \otimes_{B^{\text {op }}} f\right)=\kappa_{V, V}^{-1}\left(f \circ\left(\varepsilon \otimes_{B^{\text {op }}} I\right) \circ R\right) \\
= & \kappa_{V, V}^{-1}\left(f \circ\left(I \otimes_{B^{\text {op }}} \varepsilon\right)\right)=\sum_{i} \hat{f}_{i} \otimes_{B^{\text {op }}} f\left(\hat{v}_{i} \varepsilon(\cdot)\right)=\sum_{i} \hat{f}_{i} \otimes_{B^{\text {op }}} \varepsilon\left(f\left(\hat{v}_{i}\right) \cdot\right) \\
& =\sum_{i} \hat{f}_{i} \otimes_{B^{\text {op }}} f\left(\hat{v}_{i}\right)^{\text {op }} \varepsilon=f \otimes_{B^{\text {op }}} \varepsilon
\end{aligned}
$$


and

$$
\begin{aligned}
R^{\dagger}\left(f \otimes_{B^{\text {op }}} \varepsilon\right) & =\kappa_{V, V}^{-1} \circ R^{*} \circ \kappa_{V, V}\left(f \otimes_{B^{\text {op }}} \varepsilon\right)=\kappa_{V, V}^{-1}\left(\varepsilon \circ\left(f \otimes_{B^{\text {op }}} I\right) \circ R\right) \\
=\kappa_{V, V}^{-1}\left(f \circ\left(I \otimes_{B^{\text {op }}} \varepsilon\right) \circ R\right)=\kappa_{V, V}^{-1}\left(f \circ\left(\varepsilon \otimes_{B^{\text {op }}} I\right)\right) & =\sum_{i} \hat{f}_{i} \otimes_{B^{\text {op }}} f\left(\varepsilon\left(\hat{v}_{i}\right) \cdot\right) \\
& =\sum_{i} \hat{f}_{i} \otimes_{B^{\text {op }}} \varepsilon\left(\hat{v}_{i}\right)^{\text {op }} f=\varepsilon \otimes_{B^{\text {op }}} f
\end{aligned}
$$

Furthermore, for all $x=f \otimes_{B^{\text {op }}} g \in V^{*} \otimes_{B^{\text {op }}} V^{*}$,

$$
\begin{gathered}
\left(e^{\dagger} \otimes_{B^{\text {op }}} I\right) \circ R^{\dagger}(x)=\left(e^{\dagger} \otimes_{B^{\text {op }}} I\right) \circ \kappa_{V, V}^{-1} \circ R^{*} \circ \kappa_{V, V}(x)=\left(e^{\dagger} \otimes_{B^{\text {op }}} I\right) \circ \kappa_{V, V}^{-1}\left(\kappa_{V, V}(x) \circ R\right) \\
=\left(e^{\dagger} \otimes_{B^{\text {op }}} I\right)\left(\sum_{i} \hat{f}_{i} \otimes_{B^{\text {op }}} \kappa_{V, V}(x) \circ R\left(\hat{v}_{i} \otimes_{B} \cdot\right)=\sum_{i} \hat{f}_{i}(e)^{\mathrm{op}} \kappa_{V, V}(x) \circ R\left(\hat{v}_{i} \otimes_{B} \cdot\right)\right. \\
=\sum_{i} \kappa_{V, V}(x) \circ R\left(\hat{v}_{i} \otimes_{B} \hat{f}_{i} \cdot\right)=\kappa_{V, V}(x) \circ R\left(e \otimes_{B} \cdot\right)=\kappa_{V, V}\left(\cdot \otimes_{B} e\right)=g(f(\cdot) e)=g(e) f(\cdot) \\
=f g(e)^{\text {op }}=\left(I \otimes_{B^{\text {op }}} e^{\dagger}\right)\left(f \otimes_{B^{\text {op }}} g\right)=\left(I \otimes_{B^{\text {op }}} e^{\dagger}\right)(x)
\end{gathered}
$$

and

$$
\begin{gathered}
\left(I \otimes_{B^{\text {op }}} e^{\dagger}\right) \circ R^{\dagger}(x)=\left(I \otimes_{B^{\text {op }}} e^{\dagger}\right) \circ \kappa_{V, V}^{-1} \circ R^{*} \circ \kappa_{V, V}(x)=\left(I \otimes_{B^{\text {op }}} e^{\dagger}\right) \circ \kappa_{V, V}^{-1}\left(\kappa_{V, V}(x) \circ R\right) \\
=\left(I \otimes_{B^{\text {op }}} e^{\dagger}\right)\left(\sum_{i} \hat{f}_{i} \otimes_{B^{\text {op }}} \kappa_{V, V}(x) \circ R\left(\hat{v}_{i} \otimes_{B} \cdot\right)\right)=\sum_{i} \hat{f}_{i} \kappa_{V, V}(x) \circ R\left(\hat{v}_{i} \otimes_{B} e\right)^{\mathrm{op}} \\
=\sum_{i} \kappa_{V, V}(x) \circ R\left(\hat{v}_{i} \otimes_{B} e\right) \hat{f}_{i}(\cdot)=\sum_{i} \kappa_{V, V}(x) \circ R\left(\hat{v}_{i} \otimes_{B} \hat{f}_{i}(\cdot) e\right)=\kappa_{V, V}(x) \circ R\left(\cdot \otimes_{B} e\right) \\
=\kappa_{V, V}(x)\left(e \otimes_{B} \cdot\right)=g(f(e) \cdot)=f(e)^{\mathrm{op}} g=\left(e^{\dagger} \otimes_{B^{\text {op }}} I\right)(x)
\end{gathered}
$$

Hence $\left(V^{*}, R^{\dagger}, \varepsilon, e^{\dagger}\right) \in$ r.f.g.pYB $\operatorname{str}_{B^{\text {op }}}$.

Morphism $\kappa:() \rightarrow()^{* *}$ is natural in ${ }_{B} \mathcal{M}_{B}$, and as $V$ is finitely generated projective, $\kappa_{V}$ is invertible. Therefore it suffices to prove that $\kappa_{V}$ is a morphism in r.f.g.pYB $\operatorname{str}_{B}$. To this end, observe first that

$$
\kappa_{V}(e)=f \mapsto f(e)^{\mathrm{op}}=e^{\dagger}
$$

and, for all $v \in V$,

$$
\varepsilon^{\dagger} \circ \kappa_{V}(v)=\kappa_{V}(v)(\varepsilon)^{\mathrm{op}}=\varepsilon(v)^{\mathrm{opop}}=\varepsilon(v)
$$

Note that $\hat{f}_{i} \in V^{*}, \kappa_{V}\left(\hat{v}_{i}\right) \in V^{* *}, i \in I$ is a dual basis of $V^{*}$. Therefore, for all $\Gamma \in\left(V^{*} \otimes_{B^{\text {op }}} V^{*}\right)^{*}$,

$$
\kappa_{V^{*}, V^{*}}(\Gamma)=\sum_{i} \kappa_{V}\left(\hat{v}_{i}\right) \otimes_{B} \Gamma\left(\hat{f}_{i} \otimes_{B^{\mathrm{op}}} \cdot\right)
$$


and so, for all $v, v^{\prime} \in V$,

$$
\begin{gathered}
R^{\dagger \dagger}\left(\kappa_{V} \otimes_{B} \kappa_{V}\right)\left(v \otimes_{B} v^{\prime}\right)=\kappa_{V^{*}, V^{*}}^{-1} \circ R^{\dagger *} \circ \kappa_{V^{*}, V^{*}}\left(\kappa_{V}(v) \otimes_{B} \kappa_{V}\left(v^{\prime}\right)\right) \\
=\kappa_{V^{*}, V^{*}}^{-1}\left(\kappa_{V^{*}, V^{*}}\left(\kappa_{V}(v) \otimes_{B} \kappa_{V}\left(v^{\prime}\right)\right) \circ \kappa_{V, V}^{-1} \circ R^{*} \circ \kappa_{V, V}\right) \\
=\kappa_{V^{*}, V^{*}}^{-1}\left(\kappa_{V^{*}, V^{*}}\left(\kappa_{V}(v) \otimes_{B} \kappa_{V}\left(v^{\prime}\right)\right) \circ \kappa_{V, V}^{-1} \circ\left(x \mapsto \kappa_{V, V}(x) \circ R\right)\right. \\
=\kappa_{V^{*}, V^{*}}^{-1}\left(\kappa_{V^{*}, V^{*}}\left(\kappa_{V}(v) \otimes_{B} \kappa_{V}\left(v^{\prime}\right)\right) \circ\left(x \mapsto \sum_{i} \hat{f}_{i} \otimes_{B^{\text {op }}} \kappa_{V, V}(x) \circ R\left(\hat{v}_{i} \otimes_{B} \cdot\right)\right)\right. \\
=\kappa_{V^{*}, V^{*}}^{-1}\left(x \mapsto \sum_{i} \kappa_{V}\left(v^{\prime}\right)\left(\kappa_{V}(v)\left(\hat{f}_{i}\right) \kappa_{V, V}(x) \circ R\left(\hat{v}_{i} \otimes_{B} \cdot\right)\right)\right) \\
=\kappa_{V^{*}, V^{*}}^{-1}\left(x \mapsto \sum_{i} \kappa_{V}\left(v^{\prime}\right)\left(\hat{f}_{i}(v)^{\mathrm{op}} \kappa_{V, V}(x) \circ R\left(\hat{v}_{i} \otimes_{B} \cdot\right)\right)\right) \\
=\kappa_{V^{*}, V^{*}}^{-1}\left(x \mapsto \kappa_{V}\left(v^{\prime}\right)\left(\kappa_{V, V}(x) \circ R\left(v \otimes_{B} \cdot\right)\right)\right. \\
=\kappa_{V^{*}, V^{*}}^{-1}\left(x \mapsto \kappa_{V, V}(x) \circ R\left(v \otimes_{B} v^{\prime}\right)^{\mathrm{op}}\right) \\
=\sum_{i} \kappa_{V}\left(\hat{v}_{i}\right) \otimes_{B} \kappa_{V, V}\left(\hat{f}_{i} \otimes_{B^{\text {op }}} \cdot\right) \circ R\left(v \otimes_{B} v^{\prime}\right)^{\mathrm{op}} \\
=\sum_{i} \kappa_{V}\left(\hat{v}_{i}\right) \otimes_{B} \kappa_{V}\left(\left(\hat{f}_{i} \otimes_{B^{\text {op }}} I\right) \circ R\left(v \otimes_{B} v^{\prime}\right)\right) \\
=\left(\kappa_{V} \otimes_{B} \kappa_{V}\right)\left(\sum_{i} \hat{v}_{i} \otimes_{B}\left(\hat{f}_{i} \otimes_{B^{\text {op }}} I\right) \circ R\left(v \otimes_{B} v^{\prime}\right)\right) \\
=\left(\kappa_{V} \otimes_{B} \kappa_{V}\right) \circ R\left(v \otimes_{B} v^{\prime}\right)
\end{gathered}
$$

Therefore, $\kappa_{V}$ is a morphism in r.f.g.p $\mathrm{YB} \operatorname{str}_{B}$ as required.

Proposition 3.7 Let $\mathcal{R} \in$ r.f.g.pRge ${ }_{B}, \mathcal{C} \in$ r.f.g.pCrg . Then $(F \mathcal{R})^{*}=G\left(\mathcal{R}^{*}\right),(G \mathcal{C})^{*}=F\left(\mathcal{C}^{*}\right)$, i.e.,

$$
\begin{gathered}
\left(\mathcal{R}^{*}, \phi_{\mathcal{R}}^{\dagger}, 0,1_{\mathcal{R}}^{\dagger}\right)=\left(\mathcal{R}^{*}, \psi_{\mathcal{R}^{*}}, 0, \varepsilon^{\mathcal{R}^{*}}\right) \\
\left(\mathcal{C}^{*}, \psi_{\mathcal{C}^{\dagger}}, \varepsilon^{\mathcal{C}}, 0\right)=\left(\mathcal{C}^{*}, \phi_{\mathcal{C}^{*}}, 1_{\mathcal{C}^{*}}, 0\right)
\end{gathered}
$$

Proof. From Lemma 2.4 we know that $1_{\mathcal{R}}^{\dagger}=\varepsilon^{\mathcal{R}^{*}}$ and $1_{\mathcal{C}^{*}}=\varepsilon^{\mathcal{C}}$. Furthermore, for all $c, c^{\prime} \in \mathcal{R}^{*}$,

$$
\begin{gathered}
\phi_{\mathcal{R}}^{\dagger}\left(c \otimes_{B^{\text {op }}} c^{\prime}\right)=\kappa_{\mathcal{R}, \mathcal{R}}^{-1} \circ \phi_{\mathcal{R}}{ }^{*} \circ \kappa_{\mathcal{R}, \mathcal{R}}\left(c \otimes_{B^{\text {op }}} c^{\prime}\right) \\
=\kappa_{\mathcal{R}, \mathcal{R}}^{-1}\left(r \otimes_{B} r^{\prime} \mapsto \kappa_{\mathcal{R}, \mathcal{R}}\left(c \otimes_{B^{\text {op }}} c^{\prime}\right)\left(r r^{\prime} \otimes_{B} 1_{\mathcal{R}}+1_{\mathcal{R}} \otimes_{B} r r^{\prime}-r \otimes_{B} r^{\prime}\right)\right. \\
=\kappa_{\mathcal{R}, \mathcal{R}}^{-1}\left(r \otimes_{B} r^{\prime} \mapsto c^{\prime}\left(c\left(r r^{\prime}\right) 1_{\mathcal{R}}\right)+c^{\prime}\left(c\left(1_{\mathcal{R}}\right) r r^{\prime}\right)\right)-c \otimes_{B^{\text {op }}} c^{\prime} \\
=\kappa_{\mathcal{R}, \mathcal{R}}^{-1}\left(r \otimes_{B} r^{\prime} \mapsto c^{\prime}\left(1_{\mathcal{R}}\right) c\left(r r^{\prime}\right)+c^{\prime}\left(c\left(1_{\mathcal{R}}\right) r r^{\prime}\right)\right)-c \otimes_{B^{\text {op }}} c^{\prime} \\
=\kappa_{\mathcal{R}, \mathcal{R}}^{-1}\left(r \otimes_{B} r^{\prime} \mapsto c\left(r r^{\prime}\right)\right) c^{\prime}\left(1_{\mathcal{R}}\right)+c\left(1_{\mathcal{R}}\right)^{\text {op }} \kappa_{\mathcal{R}, \mathcal{R}}^{-1}\left(r \otimes_{B} r^{\prime} \mapsto c^{\prime}\left(r r^{\prime}\right)\right)-c \otimes_{B^{\text {op }}} c^{\prime} \\
=\left(\Delta^{\mathcal{R}^{*}} \otimes_{B^{\text {op }}} \varepsilon^{\mathcal{R}^{*}}+\varepsilon^{\mathcal{R}^{*}} \otimes_{B^{\text {op }}} \Delta^{\mathcal{R}^{*}}-I \otimes_{B^{\text {op }}} I\right)\left(c \otimes_{B_{\text {op }}} c^{\prime}\right) \\
=\psi_{\mathcal{R}^{*}}\left(c \otimes_{B^{\text {op }}} c^{\prime}\right)
\end{gathered}
$$


Similarly, for all $r, r^{\prime} \in \mathcal{C}^{*}, r r^{\prime}=\kappa_{\mathcal{C}, \mathcal{C}}\left(r \otimes_{B^{\text {op }}} r^{\prime}\right) \circ \Delta^{\mathcal{C}}$, therefore for all $r, r^{\prime} \in \mathcal{C}^{*}$,

$$
\begin{aligned}
& \psi_{\mathcal{C}}^{\dagger}\left(r \otimes_{B^{\text {op }}} r^{\prime}\right)=\kappa_{\mathcal{C}, \mathcal{C}}^{-1} \circ \psi_{\mathcal{C}}^{*} \circ \kappa_{\mathcal{C}, \mathcal{C}}\left(r \otimes_{B^{\text {op }}} r^{\prime}\right) \\
& =\kappa_{\mathcal{C}, \mathcal{C}}^{-1}\left(\kappa_{\mathcal{C}, \mathcal{C}}\left(r \otimes_{B^{\text {op }}} r^{\prime}\right) \circ\left(\Delta^{\mathcal{C}} \otimes_{B} \varepsilon^{\mathcal{C}}+\varepsilon^{\mathcal{C}} \otimes_{B} \Delta^{\mathcal{C}}-I \otimes_{B} I\right)\right) \\
& =\kappa_{\mathcal{C}, \mathcal{C}}^{-1}\left(c \otimes_{B} c^{\prime} \mapsto \kappa_{\mathcal{C}, \mathcal{C}}\left(r \otimes_{B^{\text {op }}} r^{\prime}\right) \circ \Delta^{\mathcal{C}}(c) \varepsilon^{\mathcal{C}}\left(c^{\prime}\right)+\kappa_{\mathcal{C}, \mathcal{C}}\left(r \otimes_{B^{\text {op }}} r^{\prime}\right)\left(\varepsilon^{\mathcal{C}}(c) \Delta^{\mathcal{C}}\left(c^{\prime}\right)\right)-r \otimes_{B^{\text {op }}} r^{\prime}\right. \\
& =\kappa_{\mathcal{C}, \mathcal{C}}^{-1}\left(c \otimes_{B} c^{\prime} \mapsto\left(r r^{\prime}\right)(c) \mathcal{\varepsilon}^{\mathcal{C}}\left(c^{\prime}\right)+\left(\varepsilon^{\mathcal{C}}(c)^{\text {op }} \kappa_{\mathcal{C}, \mathcal{C}}\left(r \otimes_{B^{\text {op }}} r^{\prime}\right)\right)\left(\Delta^{\mathcal{C}}\left(c^{\prime}\right)\right)\right)-r \otimes_{B^{\text {op }}} r^{\prime} \\
& =\kappa_{\mathcal{C}, \mathcal{C}}^{-1}\left(c \otimes_{B} c^{\prime} \mapsto \varepsilon^{\mathcal{C}}\left(\left(r r^{\prime}\right)(c) c^{\prime}\right)+\kappa_{\mathcal{C}, \mathcal{C}}\left(\varepsilon^{\mathcal{C}}(c)^{\text {op }} r \otimes_{B^{\text {op }}} r^{\prime}\right) \circ \Delta^{\mathcal{C}}\left(c^{\prime}\right)\right)-r \otimes_{B^{\text {op }}} r^{\prime} \\
& =\kappa_{\mathcal{C}, \mathcal{C}}^{-1}\left(c \otimes_{B} c^{\prime} \mapsto \varepsilon^{\mathcal{C}}\left(\left(r r^{\prime}\right)(c) c^{\prime}\right)+\left(\varepsilon^{\mathcal{C}}(c)^{\text {op }} r r^{\prime}\right)\left(c^{\prime}\right)\right)-r \otimes_{B^{\text {op }}} r^{\prime} \\
& =\kappa_{\mathcal{C}, \mathcal{C}}^{-1}\left(c \otimes_{B} c^{\prime} \mapsto \varepsilon^{\mathcal{C}}\left(\left(r r^{\prime}\right)(c) c^{\prime}\right)+\left(r r^{\prime}\right)\left(\varepsilon^{\mathcal{C}}\left(c^{\prime}\right)\right)\right)-r \otimes_{B^{\text {op }}} r^{\prime} \\
& =r r^{\prime} \otimes_{B^{\text {op }}} \varepsilon^{\mathcal{C}}+\varepsilon^{\mathcal{C}} \otimes_{B^{\text {op }} r} r r^{\prime}-r \otimes_{B^{\text {op }}} r^{\prime} \\
& =\phi_{\mathcal{C}^{*}}\left(r \otimes_{B^{\text {op }}} r^{\prime}\right)
\end{aligned}
$$

This completes the proof.

Remark 3.8 Put together the statements of Theorem 3.6, Theorem 3.5, Proposition 3.6 and Proposition 3.7, can be summarized in the following diagram:

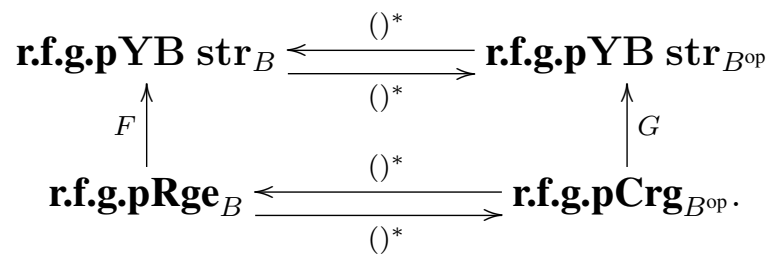

This means that the duality between right finitely generated projective ring extensions of $B$ and $B$ corings extends to the category r.f.g.pYB $\operatorname{str}_{B}$.

\section{Conclusions}

We extended the duality between right finitely generated projective ring extensions and right finitely generated projective corings to the category of right finitely generated projective generalized Yang-Baxter structures. This duality and its extension could be seen as a more general construction. For example, at the epistemologic level, the extension of the duality of (co)algebra structures seems to be a model for the relation between interdisciplinarity, pluridisciplinarity and transdisciplinarity (see [4]). It would be interesting to interpret this construction in terms of particle interactions.

The relationships between sub(co)algebras and (co)ideals are well-known, and the term of YB ideal was proposed for the first time in [11]. The following question arises: What are the relationships between sub(co)rings, (co)ideals and generalized Yang-Baxter structures?

We think that there are more connections between the Pontryagin-van Kampen duality and the above extension of the duality of (co)algebra structures. 


\section{Acknowledgments}

We would like to thank Tomasz Brzeziński for helpful remarks. The first author thanks for a Marie Curie Research Fellowship, HPMF-CT-2002-01782 at Swansea University. The work of BZ was supported by the EPSRC grant GR/S01078/01.

\section{References}

1. Morris, S.A. Pontryagin Duality and the Structure of Locally Compact Abelian Groups; Cambridge University Press: Cambridge, UK, 1977.

2. Nichita, F.F.; Schack, S.D. The duality between algebras and coalgebras. Ann. Univ. Ferrara - Sez. VII - Sc. Mat. 2005, 51, 173-181.

3. Brzeziński, T.; Wisbauer, R. Corings and Comodules; Cambridge University Press: Cambridge, UK, 2003.

4. Nichita, F.F. Algebraic models for transdisciplinarity. Transdiscipl. J. Eng. Sci. 2011, 10, 42-46.

5. Abe, E. Hopf Algebras; Cambridge University Press: Cambridge, UK, 1977.

6. Dăscălescu, S.; Năstăsescu C.; Raianu, S. Hopf Algebras: An Introduction; Marcel Dekker, Inc.: New York, NY, USA, 2000.

7. Sweedler, M.E. Hopf Algebras; W.A.Benjamin, Inc.: New York, NY, USA, 1969.

8. Sweedler, M.E. The predual theorem to the Jacobson-Bourbaki theorem. Trans. Am. Math. Soc. 1975, 213, 391-406.

9. Kassel, C. Quantum Groups; Springer Verlag: New York, NY, USA, 1995.

10. Lambe, L.; Radford, D. Introduction to the Quantum Yang-Baxter Equation and Quantum Groups: An Algebraic Approach; Kluwer Academic Publishers: Dordrecht, Germany, 1997.

11. Nichita, F.F. Non-Linear Equation, Quantum Groups and Duality Theorems. Ph.D. Thesis, The State University of New York at Buffalo, 2001.

12. Nichita, F.F. Self-inverse Yang-Baxter operators from (co)algebra structures. J. Algebra 1999, 218 , $738-759$.

(c) 2012 by the authors; licensee MDPI, Basel, Switzerland. This article is an open access article distributed under the terms and conditions of the Creative Commons Attribution license (http://creativecommons.org/licenses/by/3.0/). 